\title{
Modified everting sutures combined with reattachment to the inferior tarsal plate for involutional lower eyelid entropion: A new technique
}

\author{
Emine Malkoc Sen, Kubra Ozdemir Yalcinsoy \\ University of Health Sciences, Ulucanlar Eye Education and Research Hospital, Ankara, Turkey
}

Background This study evaluated the outcomes of a new modified Wies technique for patients with involutional lower eyelid entropion without horizontal eyelid laxity.

Methods This case series retrospectively analyzed consecutive patients with entropion who underwent surgery between January 2014 and March 2019 by the same experienced surgeon. Horizontal eyelid laxity, lower eyelid retractor function, and orbicularis muscle overriding were recorded before and after surgery. The recurrence rate and complications were also evaluated. This technique consisted of modified everting sutures combined with reattachment of the lower eyelid retractors to the inferior tarsal plate.

Results This new technique was performed on 28 eyes in 25 patients (mean age, $71.0 \pm 8.0$ years; range, $56-87$ years). Nine patients (36\%) were women and $16(64 \%)$ were men. Lower lid entropion was present in the right eye in 14 patients $(56 \%)$, the left eye in eight patients $(32 \%)$, and both eyes in three patients (12\%). The mean follow-up period was $27.3 \pm 12.4$ months (range, 6-60 months). No intraoperative complications were observed. All patients' symptoms were alleviated. One patient (3.6\%) had recurrence after 2 years (success rate, $96.4 \%$ ). The remaining 27 eyes maintained a satisfactory and comfortable eyelid position. No patients had problems with scarring.

Conclusions The approach described herein proved to be safe and feasible in eyes with involutional lower eyelid entropion without horizontal eyelid laxity. These advantages of this procedure include the lack of a conjunctival scar, punctal eversion, and lateral canthal angle deformation. A low recurrence rate and a long interval to recurrence were also observed.

Keywords Eyelids / Entropion / Modified technique / Wies procedure
Correspondence: Emine Malkoc Sen University of Health Sciences, Ulucanlar Eye Education and Research Hospital, Kale Mahallesi, Ulucanlar Caddesi, No:59, 06250, Altındağ, Ankara, Turkey Tel: $+90-505-2438124$ Fax: +90-312-3124827 E-mail: eminesentr@yahoo.com

Received: February 19, $2020 \bullet$ Revised: May 23, $2020 \bullet$ Accepted: June 18, 2020

pISSN: 2234-6163 • elSSN: 2234-6171 • https://doi.org/10.5999/aps.2020.00220 • Arch Plast Surg 2020;47:347-353

\section{INTRODUCTION}

Numerous procedures have been proposed to correct involutional entropion, but no single operative method has been es- tablished as a standard [1]. If lower lid laxity is not present, an everting suture can be placed in very elderly patients. This technique is fast, but the effect of everting sutures is temporary [2]. Other new techniques have been described, with a higher suc-

Copyright $(\odot 2020$ The Korean Society of Plastic and Reconstructive Surgeons

This is an Open Access article distributed under the terms of the Creative Commons Attribution Non-Commercial License (https://creativecommons.org/

licenses/by-nc/4.0/) which permits unrestricted non-commercial use, distribution, and reproduction in any medium, provided the original work is properly cited.

www.e-aps.org 
cess rate and lower recurrence rate than everting sutures [2-5]. We modified the Wies procedure to increase the success rate and to prevent scar formation in the conjunctiva. Our aim is to describe a modified technique for lower eyelid involutional entropion without horizontal eyelid or canthal tendon laxity and to present the findings of our technique, which combines modified everting sutures with reattachment of the lower eyelid retractors to the inferior tarsal plate.

\section{METHODS}

\section{Patients}

From January 2014 to March 2019, the author (EMS) performed this procedure in appropriate cases. A retrospective review was conducted of the charts of all patients who underwent involutional lower eyelid entropion repair at our oculoplastic department between January 2014 and March 2019. The study was approved by the ethical board (IRB No. E-19-2594) and carried out in accordance with the tenets of the Declaration of Helsinki. All patients provided written informed consent prior to enrollment.

The exclusion criteria for this procedure were horizontal eyelid laxity and medial and lateral tendon laxity. Twenty-five patients
(37.3\%) admitted for entropion surgery were eligible for this procedure. We retrospectively analyzed the medical records of these 25 patients and extracted preoperative and postoperative information on horizontal eyelid and canthal tendon laxity, lower eyelid retractor function, and orbicularis muscle overriding.

Entropion surgery was performed in 338 patients between January 2014 and March 2019. The modified entropion technique was used in 28 eyes of 25 patients who did not have horizontal eyelid and canthal tendon laxity.

Preoperative and postoperative photographs of each patient were taken and recorded in patients' electronic records. Any recorded complaints (discomfort, itching, redness, and tearing), corneal punctate staining, and photographs of each patient were evaluated before and after surgery. The recurrence rate was also investigated.

At follow-up, patients were assessed for symptom relief and resolution of the inward rotation of the eyelid margin in the primary and downward-gazing positions. Surgical success was de-fined as correct anatomical position of the eyelid and symptom relief.

\section{Steps of the surgical technique}

The skin was marked parallel to the lid edge from the punctum to the lateral canthus, $4 \mathrm{~mm}$ below the eyelashes (from the lower

\section{Fig. 1. Surgical steps of our modified technique}

Surgical steps of using modified everting sutures combined with reattachment of the retractors to the inferior tarsal plate. (A) The skin was marked parallel to the lid edge from the punctum to the lateral canthus, $4 \mathrm{~mm}$ below the eyelashes (the subtarsal skin incision). (B) The lower lid retractors (arrow). (C) Identification of the lower lid retractors (arrow). (D) Everting suture: double-armed 6.0 polyglactin sutures were passed through the midpoint of the lower lid retractors, the inferior border of the tarsal plate, and then the skin $2 \mathrm{~mm}$ inferior to the lashes.
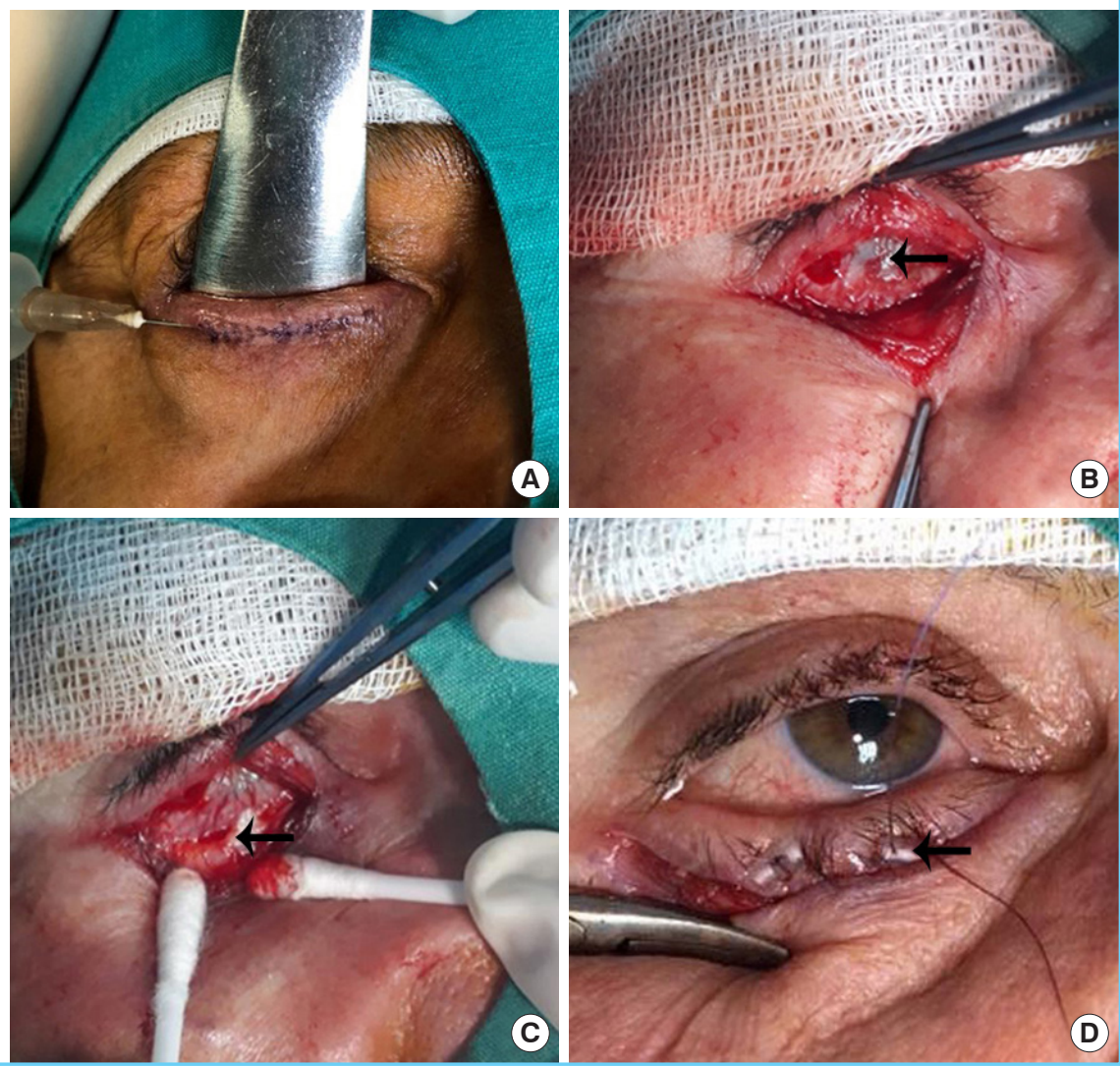
edge of the tarsus). Local anesthetic infiltration of the eyelid with lidocaine $\mathrm{HCl}$ with epinephrine was performed. A subtarsal skin incision was made with a scalpel (Fig. 1A). The orbicular muscle was carefully dissected with scissors until the lower edge of the tarsal plate was reached. It was dissected downward until the lower lid retractors were seen (Fig. 1B). A full-thickness incision was not made. The conjunctiva was not touched; it was fully preserved. First, a double-armed 6.0 polyglactin suture was passed through the midpoint of the lower lid retractors (Figs. $1 \mathrm{C}, 2 \mathrm{~A})$, and then through the inferior border of the tarsal plate in the upper wound edge (Fig. 2B), and then through the skin 2 $\mathrm{mm}$ inferior to the lashes (Fig. $2 \mathrm{C}$ ). This was first everting suture. Reattachment of the lower lid retractors to the tarsus (Fig. 2B) and placement of the everting suture (Fig. 2C) were performed in the same step. The second everting suture was placed in the same way, but laterally (Fig. 1D). If prominent orbital muscle hypertrophy was observed, 1 to $2 \mathrm{~mm}$ of skin and the orbicular muscle was horizontally excised. The skin was closed separately with interrupted 6-0 polyglactin sutures.

Skin sutures were removed in the first week. The everting sutures were left in place for 2 weeks, as they helped to form an adhesion between the lower lid retractors and inferior tarsal plate.

This technique can be classified as a modified version of the Wies technique, based on the modification of Collin, for the following reasons: (1) No full-thickness transverse skin incision was made. The conjunctiva was not touched; it was preserved. In contrast, in the Wies procedure, a full-thickness incision is made $[2,4]$; (2) The lower lid retractors were reattached to the inferior tarsal plate, instead of to the anterior tarsal plate as in the Wies procedure; (3) Two everting sutures were placed, which helped to form an adhesion between the inferior retractors and the inferior tarsal plate. A third everting suture, in the medial third of the lower lid, was not placed because it may result in punctal ectropion and epiphora. In contrast, in the Wies procedure, three everting sutures are placed $[2,4] ;(4)$ In the same step, vertical retractor laxity or disinsertion, which is the primary focus of the procedure, was corrected and the everting suture was placed; or (5) If prominent orbital muscle hypertrophy was observed, 1 to $2 \mathrm{~mm}$ of skin and the orbicular muscle was horizontally excised.

\section{RESULTS}

The mean age of the patients was $71.0 \pm 8.0$ years (range, 56-87 years). Nine of the patients (36\%) were women and 16 (64\%) were men. Lower lid entropion was present in the right eye in 14 patients (56\%), in the left eye in eight patients (32\%), and in both eyes in three patients (12\%).

All patients except one had primary involutional entropion. The one patient with a recurrent case had undergone a lateral tarsal strip (LTS) procedure 1 year ago. The mean follow-up period was $27.3 \pm 12.4$ months (range, 6-60 months). No intraoperative complications were observed.

Our technique was successful, with a satisfactory and comfortable eyelid position (Fig. 3). The symptoms of all patients were alleviated. One patient (3.6\%) experienced recurrence after 2 years. Re-operation was recommended for this patient, but the patient did not complain of the condition and chose not to undergo repeated surgery (Fig. 4). The remaining 27 eyes had a correct anatomical position of the eyelid. At every visit, patients were asked to close their eyelids, and we checked whether entropion had occurred. Problems with scarring did not occur in

\section{Fig. 2. Modified everting sutures and the retractors reattachment}

First, double-armed 6.0 polyglactin sutures were passed through the midpoint of the lower lid retractors (A), then the inferior border of tarsal plate in the upper wound edge (B), and then the skin $2 \mathrm{~mm}$ inferior to the lashes (C). Both reattachment of the lower lid retractors to the tarsus (B) and the everting suture (C) were performed in the same step.

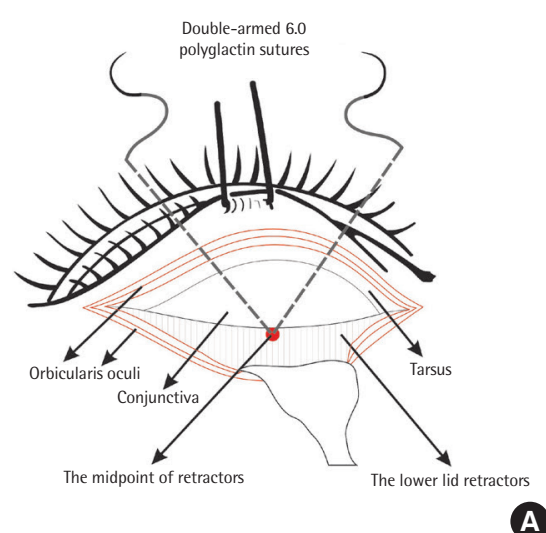

A
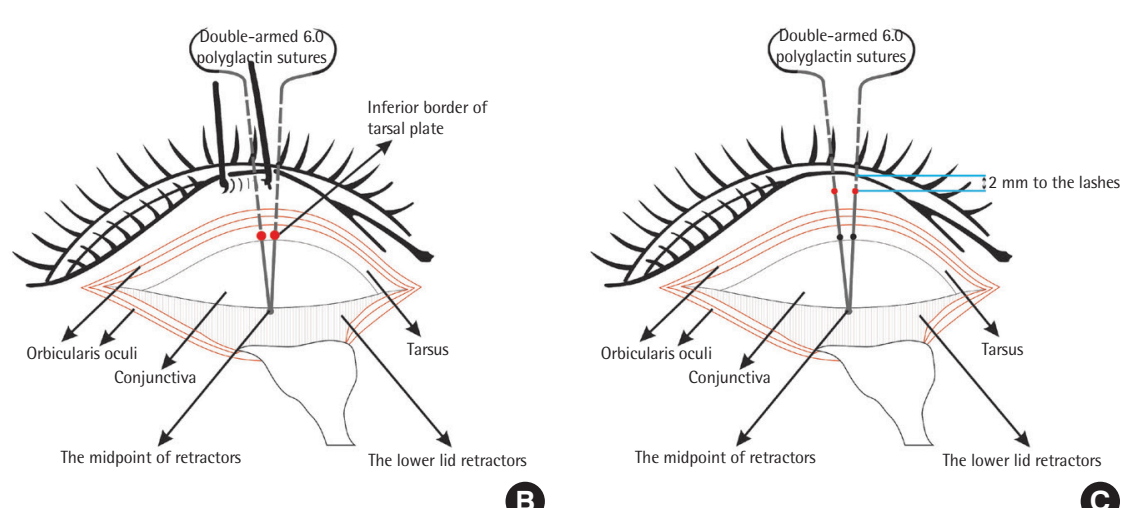
Fig. 3. Preoperative and postoperative photographs of two patients

A patient with Sturge-Weber syndrome and glaucoma. (A) Preoperative view. (B) Postoperative view at 60 months. Another patient. (C) Preoperative view. (D) Postoperative view.
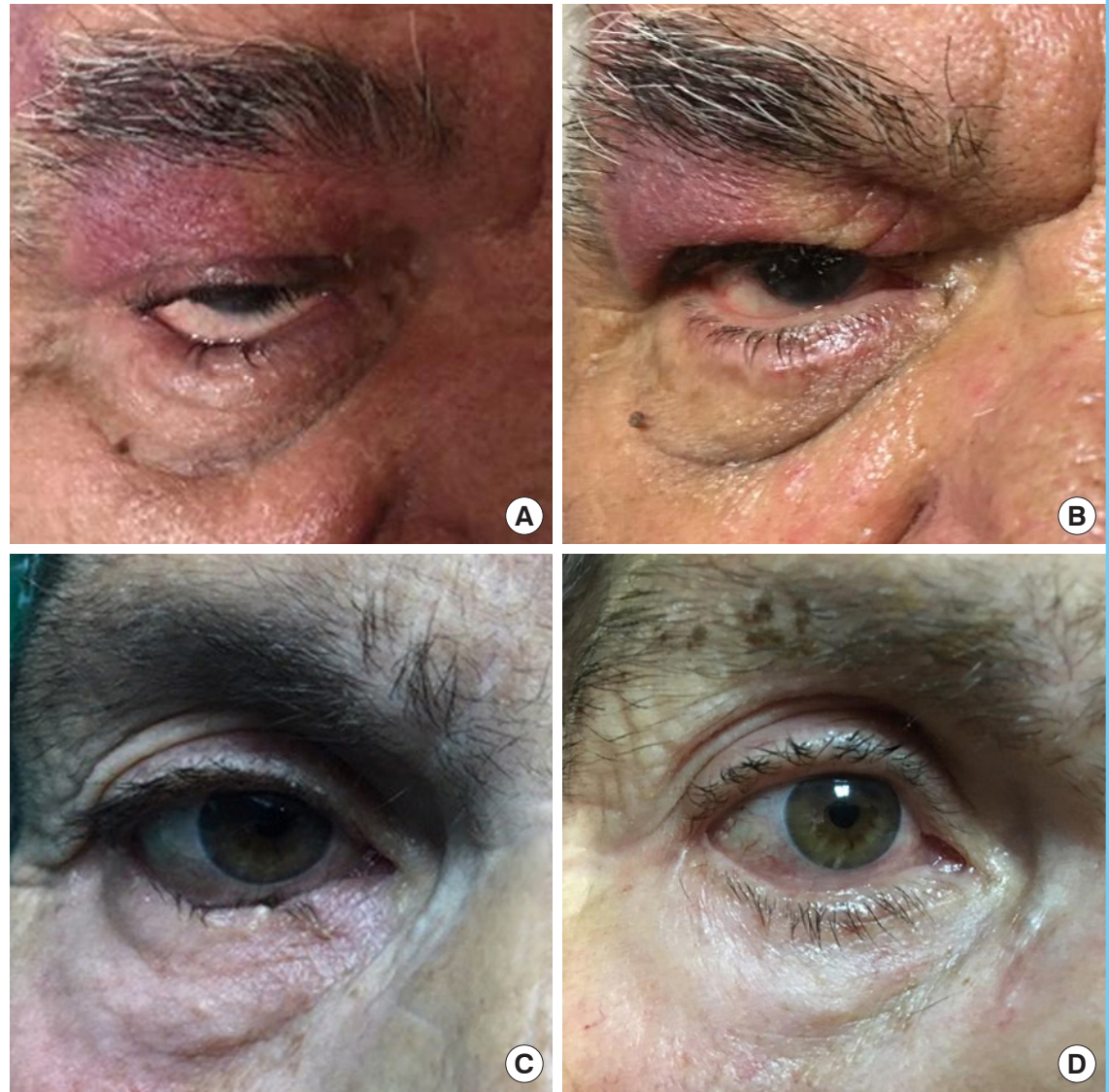

\section{Fig. 4. Photographs of recurrence in one patient}

Recurrence in one patient that underwent surgery using this technique. (A) Preoperative view. (B) Postoperative view (2 years).
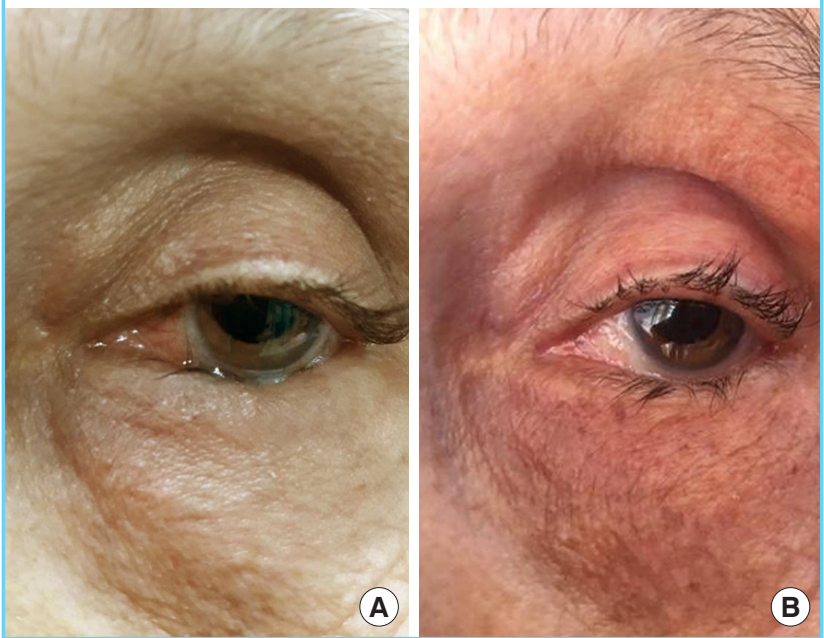

any patient, and postoperative punctal eversion or overcorrection was not observed in any cases. The success rate was $96.4 \%$ after a mean follow-up of 27 months.

\section{DISCUSSION}

Involutional lower eyelid entropion is a frequently occurring malposition of the eyelid that may threaten the eyesight [3]. The main etiological factors are disinsertion or dehiscence of the lower eyelid retractors, vertical lid laxity secondary to dehiscence, lower eyelid laxity (either of the canthal tendons or of the eyelid itself), and overriding of the preseptal orbicularis over the pretarsal orbicularis [5-8]. An ideally effective technique would be able to correct all the causative factors, while minimizing the recurrence rate [3]. The literature contains descriptions of several techniques for correcting involutional lower eyelid entropion [1,3,9-20]. Varying success and recurrence rates have been reported to result from different modified or combined entropion surgical procedures.

Everting sutures alone are known to be a safe, fast, simple, and effective treatment for involutional entropion, but the recurrence rate was $15 \%$ at a mean follow-up of 31 months $[15,16]$. If there is horizontal eyelid laxity, an everting suture can be combined with LTS to increase the efficacy of the everting suture procedure [16-18]. The recurrence rate in procedures combining everting sutures with LTS was reported to be $0 \%$ and $2 \%$ by 
Scheepers et al. [17], with a minimum follow-up of 18 months, and Barnes et al. [18], with a mean follow-up of 18 months, respectively. Differences in surgical technique or the patient population may contribute to variation in the recurrence rate [17]. However, regarding the surgical techniques combined with LTS, providing a cosmetically acceptable lateral canthal angle is one of the most important points to be considered. We think that our modified technique is especially advantageous because it does not cause lateral canthal angle deformation.

If horizontal eyelid laxity is present, the Wies method can be combined with LTS $[13,14]$. In a study by Serin et al. [13], recurrence was observed in nine of the 31 eyes $(29.0 \%)$ in which the Wies procedure was performed, whereas recurrence only was found in one of the 45 eyes (2.2\%) treated with a technique combining LTS, retractor tightening, and everting sutures. On average, recurrence took place 4.8 months after the procedure in the Wies group, while the only patient with recurrence in the combined procedure group presented at 2 months postoperatively [13]. Lance and Wilkins [14] reported a recurrence rate of $11 \%$ in 66 cases treated with the Wies procedure alone, whereas no recurrences were observed in 29 cases treated with a procedure combining the Wies procedure with lateral canthal horizontal shortening [14]. In this study, recurrence was observed in only one eye (3.6\%) at 24 months of follow-up. Therefore, we suggest that long-term, regular follow-up of patients is important for detecting long-term recurrence.

If there is no horizontal eyelid laxity, as in our patients, the Wies and Jones procedures can be considered as alternative options. The Jones technique may be used as a primary procedure, but it is mostly used for recurrent involutional entropion [2]. Athavale and O'donnell [9] reported that LTS combined with a modified version of the Jones technique (lower eyelid retractor reattachment onto the anterior surface of the tarsal plate) was an effective surgical technique based on the results of 24 months of follow-up. Because horizontal eyelid laxity might have been present, all patients underwent lateral canthoplasty and LTS as part of the procedure. Athavale and O'donnell [9] did not place everting sutures. In their technique, the sutures were passed through the inferior skin border, then through retractors and anterior surface of tarsal plate, and then through the upper edge of the skin border. Our procedure involved modified everting sutures combined with direct reattachment to the inferior tarsal plate of the lower eyelid retractors. In our procedure, the sutures were passed first through the lower lid retractors, and then the inferior border of the tarsal plate, and then through skin $2 \mathrm{~mm}$ inferior to the lashes (the first everting suture). The skin was closed separately with interrupted 6-0 polyglactin sutures.

The Wies procedure mainly corrects vertical laxity and orbicu- laris dysfunction $[2,4,13]$. It is a combination of transverse fullthickness blepharotomy and everting sutures $[2,4,13]$. In our technique, a full-thickness incision is not done. Therefore, the conjunctiva is preserved. This point is very important, particularly in patients with ocular cicatricial pemphigoid (OCP). Although the etiology is not involutional, entropion is a common finding in OCP patients. This technique may also be useful in patients with OCP.

If horizontal eyelid laxity is not present, we suggest that the Wies procedure can be modified to increase the success rate. In this way, it may be more effective than the standard Wies procedure. The recurrence rates after the Wies procedure have been reported to be $11 \%-29 \%[13,14,18,19]$. In our modified Wies technique, the rate of recurrence was $3.6 \%$ and the time to recurrence was 2 years in the sole patient who experienced recurrence. Another advantage of this method may be that recurrence occurs later than in other methods reported in the literature [124]. The cause of late recurrence may be fibrosis between the tarsus and retractors. This fibrosis may also prevent overriding and relapse in the late period. Furthermore, both strengthening the retractors of the lower eyelid and making the everting sutures in the same step shortened the surgery time. Moreover, the conjunctiva and fornix were preserved, the effectiveness of the Wies procedure was increased, and the rate of complication was dramatically reduced with this method. Another advantage is that the lateral canthal angle did not deteriorate. As we know, the cosmetic results of a surgical technique should be taken into consideration when selecting the most appropriate procedure, and cosmetic results may be as important as surgical outcomes in oculoplastic surgery. No patients had problems with scarring or the lateral canthal angle in our study. However, our study lacks a comparison with standard everting sutures, the Wies procedure, or the Jones procedure. The retrospective nature of our study and limited number of patients are other limitations of our research. Although many studies investigating surgical repair techniques for involutional entropion have compared combined or modified techniques with more traditional ones [1$24]$, to our best knowledge, this particular modification has not been described in the literature.

A recently published review analyzed studies examining various causative factors and treatment procedures to clarify and correct involutional lower eyelid entropion [20]. Using a combination of these treatment procedures to correct multiple etiological factors further decreases the recurrence rate, as in our modified technique [20]. In our study, a patient with recurrent entropion after LTS was successfully treated with this method. Additionally, the recurrence rate in our study (3.6\%) was low.

We propose that this modified Wies technique (transverse 
semi-thickness blepharotomy and modified everting sutures combined with reattachment of the lower eyelid retractors to the inferior tarsal plate) is an effective surgical procedure that may be preferred for the primary treatment of involutional lower entropion. The advantages of this procedure include the lack of a conjunctival scar, punctal eversion, and lateral canthal angle deformation. Additionally, a low recurrence rate and a long interval until recurrence were observed. We believe that our technique might be very useful in appropriate cases.

\section{NOTES}

\section{Conflict of interest}

No potential conflict of interest relevant to this article was reported.

\section{Ethical approval}

The study was approved by the Institutional Review Board of University of Health Sciences, Numune Education and Research Hospital (IRB No. E-19-2594) and performed in accordance with the principles of the Declaration of Helsinki. Written informed consents were obtained.

\section{Patient consent}

The patients provided written informed consent for the publication and the use of their images.

\section{Author contribution}

Data curation: KO Yalcinsoy. Formal analysis: EM Sen. Methodology: EM Sen. Writing - original draft: EM Sen. Writing - review \& editing: EM Sen, KO Yalcinsoy.

\section{ORCID}

Emine Malkoc Sen https://orcid.org/0000-0002-5373-8987 Kubra Ozdemir Yalcinsoy

$$
\text { https://orcid.org/0000-0002-3352-9547 }
$$

\section{REFERENCES}

1. Asamura S, Kakizaki H, Shindou E, et al. What is the best strategy for Asians with involutional entropion? J Craniofac Surg 2014;25:972-5.

2. Tyers AG, Collin JRO. Colours atlas of ophthalmic plastic surgery. London: Elsevier; 2008.

3. Roberts MA, Baddeley P, Sinclair N, et al. The lower lid diamond: a simple entropion repair to correct both horizontal and lower-lid retractor laxity. Ophthalmic Plast Reconstr Surg 2012;28:44-6.
4. Wies FA. Spastic entropion. Trans Am Acad Ophthalmol Otolaryngol 1955;59:503-6.

5. Jones LT. The anatomy of the lower eyelid and its relation to the cause and cure of entropion. Am J Ophthalmol 1960;49: 29-36.

6. Benger RS, Musch DC. A comparative study of eyelid parameters in involutional entropion. Ophthalmic Plast Reconstr Surg 1989;5:281-7.

7. Hurwitz JJ. Senile entropion: the importance of eyelid laxity. Can J Ophthalmol 1983;18:235-7.

8. Kakizaki H, Chan WO, Takahashi Y, et al. Overriding of the preseptal orbicularis oculi muscle in Caucasian cadavers. Clin Ophthalmol 2009;3:243-6.

9. Athavale DD, O'donnell BA. Lower eyelid entropion repair with retractor mobilization and insertion onto the anterior surface of the tarsal plate. Orbit 2018;37:121-4.

10. Kreis AJ, Shafi F, Madge SN. Transconjunctival entropion repair: the backdoor approach. Orbit 2013;32:271-4.

11. Nakauchi K, Mimura O. Combination of a modified Hotz procedure with the Jones procedure decreases the recurrence of involutional entropion. Clin Ophthalmol 2012;6: 1819-22.

12. Cheung JJC, Wong CKH, Cheung LTY. Combined pentagonal resection and inferior retractor plication in involutional entropion. BMC Ophthalmol 2018;18:329.

13. Serin D, Buttanri IB, Karslioglu S, et al. The efficacy of the combined procedure in involutional entropion surgery: a comparative study. Korean J Ophthalmol 2013;27:405-8.

14. Lance SE, Wilkins RB. Involutional entropion: a retrospective analysis of the Wies procedure alone or combined with a horizontal shortening procedure. Ophthalmic Plast Reconstr Surg 1991;7:273-7.

15. Wright M, Bell D, Scott C, et al. Everting suture correction of lower lid involutional entropion. Br J Ophthalmol 1999; 83:1060-3.

16. Mocan MC, Erdener U, Irkec M, et al. Evaluation of two surgical procedures for the management of intermittent involutional entropion. Turk J Ophthalmol 2009;39:332-8.

17. Scheepers MA, Singh R, Ng J, et al. A randomized controlled trial comparing everting sutures with everting sutures and a lateral tarsal strip for involutional entropion. Ophthalmology 2010;117:352-5.

18. Barnes JA, Bunce C, Olver JM. Simple effective surgery for involutional entropion suitable for the general ophthalmologist. Ophthalmology 2006;113:92-6.

19. Boboridis K, Bunce C, Rose GE. A comparative study of two procedures for repair of involutional lower lid entropion. Ophthalmology 2000;107:959-61. 
20. Lin P, Kitaguchi Y, Mupas-Uy J, et al. Involutional lower eyelid entropion: causative factors and therapeutic management. Int Ophthalmol 2019;39:1895-907.

21. Guthrie AJ, Kadakia P, Rosenberg J. Eyelid malposition repair: a review of the literature and current techniques. Semin Plast Surg 2019;33:92-102.

22. Pereira MG, Rodrigues MA, Rodrigues SA. Eyelid entropion. Semin Ophthalmol 2010;25:52-8.
23. Altieri M, Iester M, Harman F, et al. Comparison of three techniques for repair of involutional lower lid entropion: a three-year follow-up study. Ophthalmologica 2003;217: 265-72.

24. Prendes MA, Geng J, Ediriwickrema LS, et al. Assessment of the safety and efficacy of prolapsed orbital fat resection during involutional entropion repair. Ophthalmic Plast Reconstr Surg 2020;36:34-7. 